\title{
What is government's role in medical apps?
}

A $\mathrm{n}$ estimated 1.5 billion smartphone owners will use medical apps by 2018. With more than 300 medical apps available on iTunes already, selecting the right one is challenging. Does government have a role to play?

The National Health Service (NHS) in the United Kingdom thinks so. It recently endorsed apps that could be used in cognitive behaviour therapy in its newly curated database. A list of all government-approved medical mobile apps, with user comments and ratings, is available on the NHS' health apps library.

"The main purpose of the health apps library is to make it simple for the public to easily find safe apps to help them manage their health," stated Murray Morse, NHS senior media relations manager, in an email to CMAJ.

Although some medical apps can be found using the search function on Health Canada's medical devices active licence listing, the information is scant, often little more than the app name. Health Canada stated in an email response that only five mobile apps are searchable through this function. The department stated that it did not have time for an interview to answer additional questions.

Like Health Canada, the US Food and Drug Administration (FDA) has a search function on its $510(\mathrm{k})$ premarket notification database for medical devices that includes all its higher-risk medical devices. However, the US database provides more information than Health Canada's, including an app's intended use and performance data. The FDA stated that it does not keep track of the exact number of apps searchable through this function. An incomplete sampling of all approved apps can be found on the FDA website, however, which currently lists 115 apps.

In addition to providing a database of approved apps to help alleviate consumer confusion, some governments also play a larger role in licensing apps. The UK, United States and Canada all use medical device regulations for

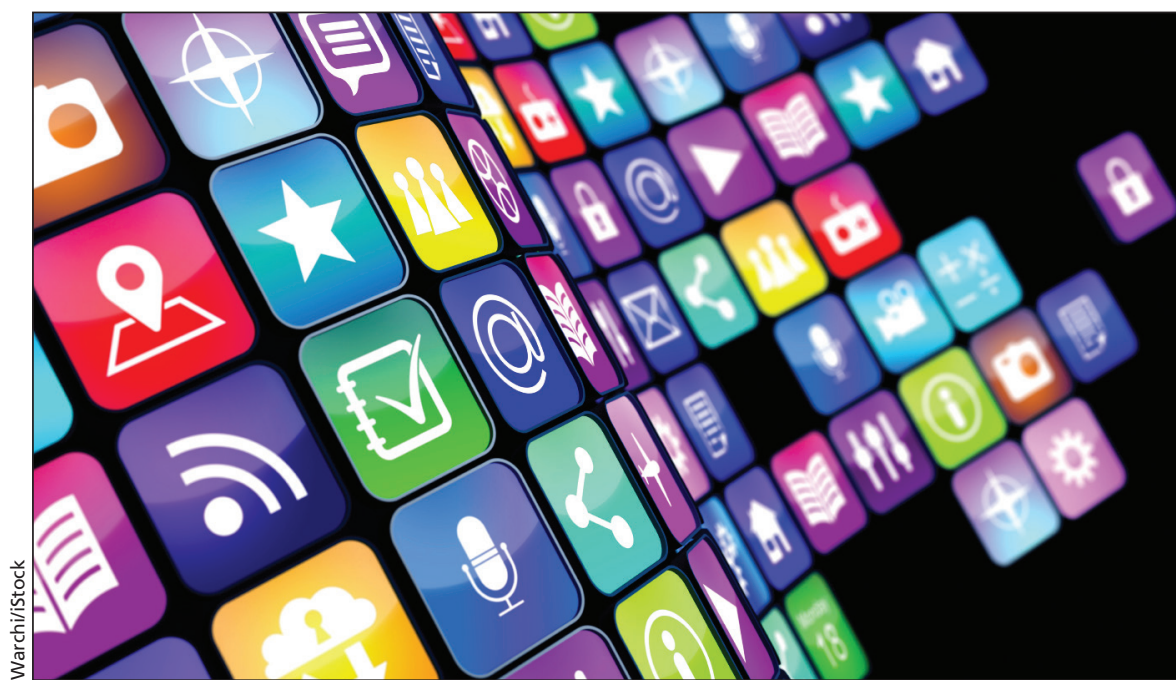

The UK government curates a site of endorsed medical apps. Should Health Canada?

licensing mobile medical apps and have similar risk-grading systems.

In Canada, a medical device licence needs to be granted by Health Canada before marketing for apps in higher-risk categories, such as those that aid in the diagnosis or treatment of medical conditions. However, lower-risk apps, such as those that monitor weight to encourage healthy living, do not require a licence. But they still must comply with regulations and are subject to enforcement by Health Canada, which could include recall from the market until a licence has been granted.

However, what is lacking in Canada and present in both the UK and US are specific guidelines about which types of mobile apps fall under each medical device category. This deficit can be confusing for some manufacturers.

Michael Zagorski, director of quality and regulatory affairs for the medical app Sway (a concussion management system) said he had difficulties. Sway was initially submitted and approved as a Class I device (low risk, no licence required). But the product was delayed, and upon resubmission it was reclassified as a Class II device (higher risk, full licence required).

"It's not black and white," says Zagorski of the classification system.
"Interpretation is required."

Tim Squire, a life sciences regulatory lawyer and partner with Fasken Martineau, who has worked with medical apps and devices, believes that manufacturers could benefit from additional guidance. "There's nothing from Health Canada that helps provide a threshold whereby you can assess how much of a risk the app is."

When the device classification of a medical app is clear to the manufacturer, however, the application process may not be an issue. Albert Boniske, director of regulatory affairs at AliveCor, had no doubt that its mobile app AliveECG (a mobile heart monitor) should be a higherrisk medical device and was content with Health Canada's application process.

AliveECG also received approval from the NHS and the FDA; Boniske said he was content with all three agencies.

Canada is part of the International Medical Device Regulators Forum an international working group on medical devices - and an email statement from Health Canada indicated that its current guidelines may be updated as a result of this collaboration. - Wilson Kwong, Kingston, Ont.

CMAJ 2015. DOI:10.1503/cmaj.109-5063 